\title{
Analysis of Flow Field Dynamic Characteristics of Friction Pair Fluid-Film for Heat Pump Mechanical Seal
}

\author{
Mutellip Ahmat ${ }^{1, a^{*}, \text { Ning Li }}{ }^{2, b}$ \\ ${ }^{1}$ School of Electrical Engineering, Xinjiang University, Urumqi, 830047, Xinjiang, China \\ ${ }^{2}$ School of Mechanical Engineering, Xinjiang University, Urumqi, 830047, Xinjiang, China \\ amtlp@xju.edu.cn, ${ }^{\mathrm{b}}$ ningli_520@126.com
}

\begin{abstract}
Keywords: Mechanical seal; Friction pair interface; Fluid-film flow field; Dynamic characteristics; CFD;

Abstract. The gap of the friction pair interface is tiny for the heat pump mechanical seal, the dynamic characteristics of the fluid-film couldn't be come true easily when using the traditional experimental test. In this paper, the 3D FEM model of the fluid-film is set up, and then the dynamic characteristics of the fluid-film were simulated by the computational fluid dynamics (CFD) theory and method, the pressure of the fluid-film were gotten, and the results of the analysis were compared with the theoretical values to get a better agreement. Then based on this model, the impact laws of the differential pressure and the width on the pressure were further studied, the results provide a useful reference for the design of heat pump mechanical seal.
\end{abstract}

\section{Introduction}

Heat pump mechanical seal system is a kind of mechanical seal type that was commonly used in the petroleum and chemical industry; it is also an important studying object for mechanical seals with the high parameters (high temperature, high pressure and high speed)and the extremely working condition[1]. When the heat pump mechanical seal operates, the pressure that the fluid-film formed is non-linear. When the pressure is transmitted to the seal ring, it will caused the force uniform, resulting the deformation of the sealing ring, then caused the changes in the thickness of the liquid film, so as to increase the amount of leakage, and even the seal failure; therefore, it is necessary to study the dynamic characteristics of the fluid-film and the influence of operating parameters on the pressure.

In this research, the numerical simulation was carried to the fluid-film for the dynamic characteristics by based on CFD, then the pressure of fluid-film was obtained by solving the three-dimensional N-S equations, and the accuracy of numerical simulation was verified by compared the numerical simulation with the theoretical results, and the relationship between the working conditions and pressure was also speculated. The researching results provided a scientific basis for the optimization designing of the high parameter heat pump mechanical seal.

\section{The Numerical Calculation Theory}

The CFD numerical simulation theory and method mainly contains the following three basic equations: the momentum conservation, the energy conservation and the mass conservation equation [2]. But they are hard to get the analytical solution. In order to solve these equations, these integral or partial differential equation were replaced by the discrete algebraic equation. Firstly, the above equations were discrete, and then the basic physicals (such as speed, pressure and density, etc.) in the flow field were obtained through solving these established algebraic equations that reflected the variable relationship at the discrete points [3].

In the process of theoretical analysis and derivation, the hypothesis was made according to the flow characteristics of fluid-film:

(1) The fluid-film is continued and laminar; the small part of the humble was ignored. 
(2) The fluid-film is Newton's viscosity, no slipping.

(3) The fluid flow is steady state.

The dynamic characteristics were studied by based on the Navier-Stock and continuity equations.

The continuity equations :

$\frac{\partial(\rho)}{\partial t}+\frac{\partial(\rho u)}{\partial x}+\frac{\partial(\rho v)}{\partial y}+\frac{\partial(p w)}{\partial z}=0$

Based on the above assumption, the Navier-Stokes equations simplified shown as following [4]:

$$
\left\{\begin{array}{l}
\frac{\partial p}{\partial x}=\mu \frac{\partial^{2} w_{x}}{\partial z^{2}} \\
\frac{\partial p}{\partial y}=\mu \frac{\partial^{2} w_{y}}{\partial z^{2}} \\
\frac{\partial p}{\partial y}=0
\end{array}\right.
$$

Where $\rho$ is the density, $\mathrm{t}$ is time, $\mathrm{u}$ is the velocity vector, $\mathrm{u}, \mathrm{v}$ and $\mathrm{w}$ is the velocity vector in the $\mathrm{x}$, $\mathrm{y}$ and $\mathrm{z}$ direction component.

The theoretical formula of pressure of the fluid-film was deduced by the above assumptions.

The velocity boundary of surface shown as following:

$$
\begin{aligned}
& Z=0, w_{X}=U_{1}, w_{y}=V_{1}, w_{Z}=0 \\
& Z=h, w_{X}=U_{2}, w_{y}=V_{2}, w_{Z}=W
\end{aligned}
$$

The theoretical calculation formula of the carrying capacity and the pressure of the fluid-film were deduced by based on the above assumptions and boundary conditions.

The pressure distribution formula shown as following:

$$
P(r)=\frac{p_{0}-p_{i}}{\ln \frac{r_{0}}{r_{i}}} \ln (r)+\frac{p_{i} \ln \left(r_{0}\right)-p_{0} \ln \left(r_{i}\right)}{\ln \frac{r_{0}}{r_{i}}}
$$

Where $\mathrm{P}_{0}$ and $\mathrm{P}_{\mathrm{i}}$ is respectively the inlet and outlet pressure of the fluid-film, ro and ri is respectively the outer and inner diameter of the fluid-film, $\mathrm{F}$ is the carrying capacity of the fluid -film, $\mathrm{P}(\mathrm{r})$ is the pressure of the point which diameter is $\mathrm{r}$.

\section{The Numerical and Theoretical Calculation to the Fluid Film}

\section{Computed method}

In the research of the numerical simulation of the fluid-film, firstly the continuous physical fields in spatial domain were replaced, such as the velocity and the pressure fields, with a set of a finite number of variable values at discrete points, and then the algebraic equations about the relationship between these variables field variables at discrete points were established[5]. Finally, these algebraic equations were solved to obtain an approximation of the field variables. To establish a discrete control equation in the spatial domain, the grid must be used; the different numerical solution needs different type of grid. After meshing, the initial and boundary conditions were identified. When they were assigned to the appropriate node, it still needs to specify fluid physical parameters and control parameters.

\section{The Dynamic Characteristics of the Fluid-Film}

\section{Fluid-Film Modeling}

In this paper, a kind of Heat pump mechanical seal was used to study. Firstly the three-dimensional numerical modeling of the fluid-film was build up by based on actual condition. The geometric model is shown in Fig.1. 

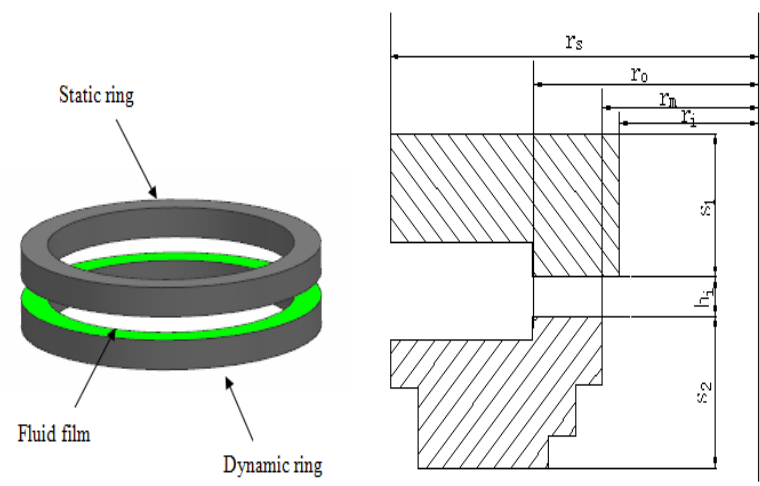

Fig.1 The fluid-film model of sealing ring

The model includes dynamic ring, static ring, fluid medium between the two rings. Where, $\mathrm{r}_{\mathrm{m}}$ and $r_{s}$ are respectively the inner and outer radius of the seal ring, $S_{1}$ and $S_{2}$ are respectively the thickness of static and dynamic ring; $h_{i}$ is the end clearance of inner diameter.

\section{Computational model}

The sealing end face of mechanical seal is periodicity in the circumferential direction, so the dynamic characteristics of the fluid-film are also periodic [6]. In order to reduce the amount of computation, 1/4 of the fluid-film model is used as the calculate zone, the obtained results are also single cycle, and then the calculation result is extended to the whole cycle. Computational models are shown in Fig.2.

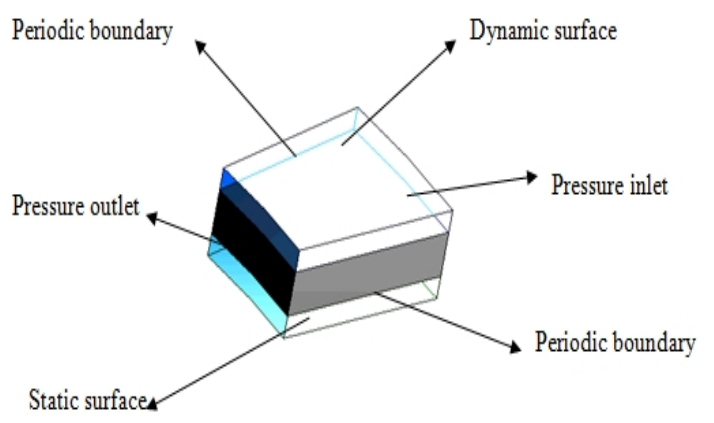

Fig. 2 The computational models

\section{The Pressure of the Fluid-film}

This study is mainly based on the working-environment parameters of mechanical seals to simulated and gain flow field characteristics of the fluid-film. The material properties of fluid-film were showed in Tab.1. The boundary conditions were showed in Tab.2.

Tab.1 Material properties of the fluid medium

\begin{tabular}{lccc}
\hline Density & $\mathrm{Cp}$ & Viscosity & Thermal Conductivity \\
\hline $886 \mathrm{~kg} / \mathrm{m}$ & $1400 \mathrm{~J} / \mathrm{kg}-\mathrm{k}$ & $0.02 \mathrm{Kg} / \mathrm{m}-\mathrm{s}$ & $0.12 \mathrm{~W} / \mathrm{m}-\mathrm{K}$ \\
\hline
\end{tabular}

Tab.2 Boundary conditions of fluid-film

\begin{tabular}{|c|c|c|}
\hline Speed Inlet Pressure & Outlet Pressure & Temperature \\
\hline 1500Rpm & $0.1 \mathrm{Mpa}$ & $40 \square$ \\
\hline
\end{tabular}

According to the above settings, the flow field characteristics of the fluid-film were obtained. Fig. 3 is the numerical simulation of the fluid- film.

As can be seen from Fig.3, the pressure of fluid-film increases from the inner diameter to the outer, and the differential pressure is relatively large, which will cause the load is not uniform, thus accelerate the deformation of the sealing ring.

According to the formula (4) and the finite element program provided in the literature, the theoretical pressure values of fluid-film at each radius point were obtained. The results of the numerical simulation are compared with the analytical results. The results are shown in Fig.4. 


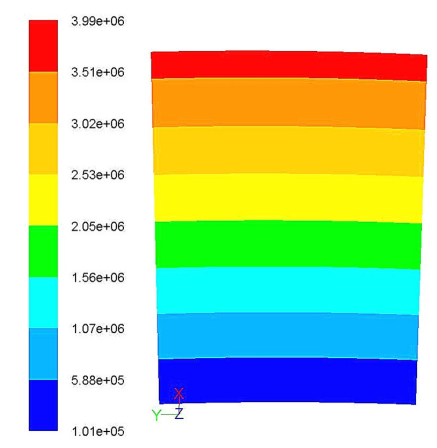

Fig.3 The Pressure field contours

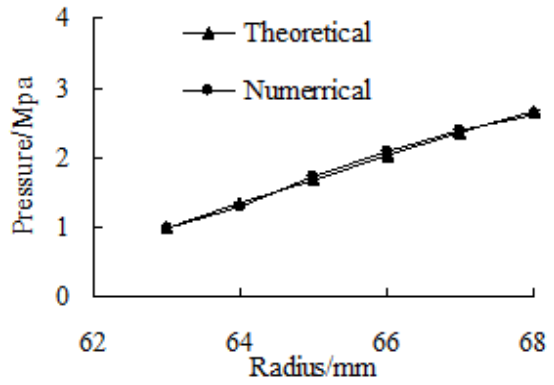

Fig.4 The theoretical calculation and the numerical simulation

From Fig.4, when the radius of the fluid-film becomes the larger, the pressure of the fluid-film gradually increases until the same pressure as the outside. The values of the theoretical and numerical cross each other, and the growth is a kind of evenly and constant slope. They are consistent; it indicated that the simulation is accuracy at a certain degree.

\section{The relationship between the pressure and the differential pressure}

The pressure has a certain relationship with different pressure of fluid-film that can be seen from equation (4). When the different pressure was $2 \mathrm{Mpa}, 4 \mathrm{Mpa}, 6 \mathrm{Mpa}, 8 \mathrm{Mpa}$, the relationship between the pressure and the different pressure is studied. The result is shown in Fig.5.

From Fig.8, at the same radial point, as the inlet pressure becomes larger or smaller, pressure of the film increase or decrease correspondingly, they were isotropic.

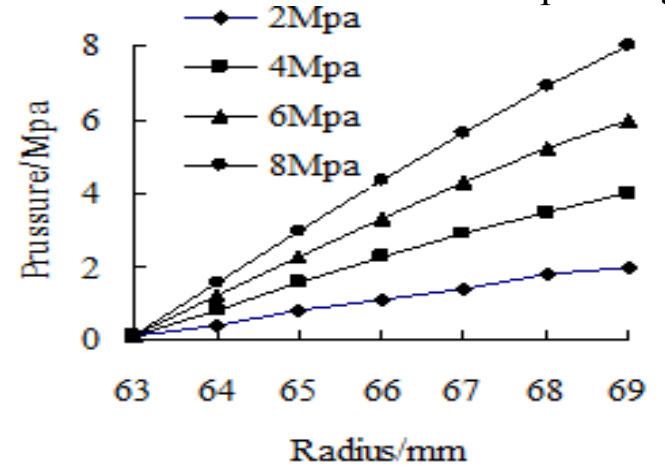

Fig.5 The differential and the fluid-film pressure

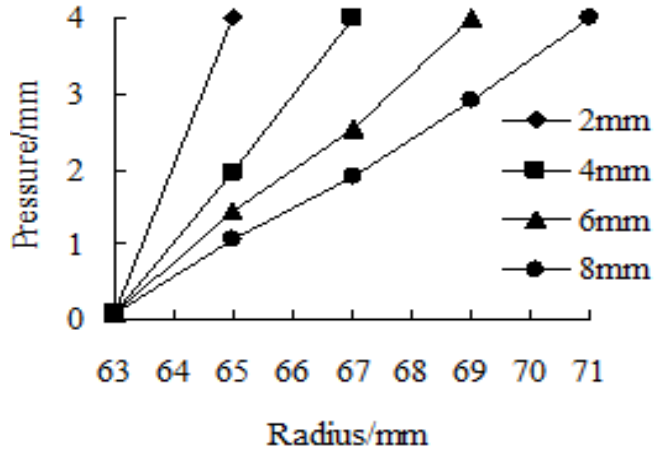

Fig. 6 The pressure with different width

\section{The relationship between the pressure and the width}

The size of width chosen are $2 \mathrm{~mm}, 4 \mathrm{~mm}, 6 \mathrm{~mm}, 8 \mathrm{~mm}$, the inlet pressure is $0.1 \mathrm{Mpa}$, outlet pressure is 4Mpa. The minimum inside diameter of the model is $63 \mathrm{~mm}$. The result of computational simulation is shown in Fig.6.

From Fig.6, when the width increases, the slope of each line is smaller; however, the rate of change is not the same. When the width is $2 \mathrm{~mm}$, the variation of the pressure is $3.9 \mathrm{Mpa}$ in every 2 radiation change. It is $1.95 \mathrm{Mpa}$ for $4 \mathrm{~mm}$, and so on, $6 \mathrm{~mm}$ and $8 \mathrm{~mm}$ is $1 / 3$ and $1 / 4$. So the pressures of $2 \mathrm{~mm}$ and $8 \mathrm{~mm}$ are listed separately to find out the relationship between the pressure and the total width.

\section{Summary}

Under the working conditions of the high speed, high pressure and high temperature, the pressure of the fluid-film changed rapidly, which would affect the sealing performance of the heat pump mechanical seal.

The pressure of the fluid-film increased with the width and the working pressure. 
The simulated result of dynamic characteristics of fluid-film in different conditions provided a scientific basis for the optimization designing of the high parameter heat pump mechanical seal.

\section{Acknowledgements}

This project is supported by National Natural Science Foundation of China (Grant No.51565056).

\section{References}

[1] Weian Meng, Mutellip Ahmat. Study on the Field Dynamics of the Seal Cavity Flow Field for High parameters Bellows Mechanical Seal [J].Applied Mechanics and Materials, 2011.

[2] Noel B , Benoit M. Heat transfer in a mechanical face seal [J]. International Journal of Thermal Sciences, 2009 , 48: 781-794.

[3] Rahul N. Samant, Patrick E. Plelan, M. Finite element analysis of residual-stress-induced flatness deviation in banded carbon seals [J]. Finite Elements in Analysis and Design, 38(2002): 785-801.

[4] Shifeng $\mathrm{Wu}$, Ray Clark. Positioning of Hydrodynamic Lift Features on Non-Contacting Mechanical Gas Seal Rings [J]. Tribology Transactions, 2000, 43: 498-506.

[5] Atta Sojoudi, Ali Mazloomi, Suvash C. Saha. Similarity Solutions for Flow and Heat Transfer of Non-Newtonian Fluid over a Stretching Surface [J]. JOURNAL OF APPLIED MATHEMATICS, 2014.

[6] Xu-dong Peng, Wei Liu. Thermo-Elasto-Hydrostatic Effect Analysis of a Double Tapered Hydrostatic Mechanical Seal in Reactor Coolant Pumps [J]. Journal of Tribology, 2012, 32 (3). 